UCRL-JC-128357

PREPRINT

Temperature and Spectral Investigation of Bulk KDP Below Damage Using 355 nm Laser Irradiation

\author{
S. G. Demos, M. Yan, M. Staggs, B. W. Woods, \\ Z. L. Wu, H. B. Radousky, J. J. De Yoreo
}

This paper was prepared for submittal to the Annual Symposium on Optical Materials for High Power Lasers Boulder, CO

October 6-8, 1997

October 1, 1997

This is a preprint of a paper intended for publication in a journal or proceedings. Since changes may be made before publication, this preprint is made available with the understanding that it will not be cited or reproduced without the permission of the author. 


\section{DISCLAIMER}

This document was prepared as an account of work sponsored by an agency of the United States Government. Neither the United States Government nor the University of California nor any of their employees, makes any warranty, express or implied, or assumes any legal liability or responsibility for the accuracy, completeness, or usefulness of any information, apparatus, product, or process disclosed, or represents that its use would not infringe privately owned rights. Reference herein to any specific commercial product, process, or service by trade name, trademark, manufacturer, or otherwise, does not necessarily constitute or imply its endorsement, recommendation, or favoring by the United States Government or the University of California. The views and opinions of authors expressed herein do not necessarily state or reflect those of the United States Government or the University of California, and shall not be used for advertising or product endorsement purposes. 


\title{
Temperature and spectral investigation of bulk KDP below damage using 355 nm laser irradiation
}

\author{
S. G. Demos, M. Yan, M. Staggs, B. W. Woods, Z. L. Wu, H. B. Radousky and J.J. De Yoreo \\ Lawrence Livermore National Laboratory, PO Box 808, Livermore, CA 94580.
}

Tel.: (510) 423 3388, Fax: (510) 4232463

\begin{abstract}
A spectral and temperature investigation of fast-grown KDP crystals under high fluence, $355 \mathrm{~nm}$ laser irradiation is discussed. Pump-and-probe Raman spectroscopy indicate transient changes of the vibrational spectrum. Photothermal deflection experiments provide information on the temporal behavior of the temperature change. The presence of emission in the visible and NIR spectral regions is attributed to the presence of impurities and/or defects in the crystal.
\end{abstract}

Key words: KDP, Raman scattering, photothermal deflection, damage

\section{INTRODUCTION}

The continuous development of high power laser systems is accompanied with the need for optical components with enhanced performance characteristics. One of the main problems is to design optical components that have increased damage threshold. In the presence of defects, the physical processes preceding laser induced damage in wide-gap optical materials are even more complex. ${ }^{1-4}$ It is currently believed by many researchers that bulk damage in nonabsorbing optical materials is initiated by the generation of free carriers which, in turn, absorb more light and lead to irreversible cascade processes.

In this work, Raman scattering, photothermal deflection and emission spectroscopy techniques are employed to study processes taking place at the sub-damage threshold intensity in fast grown KDP crystals. The experimental results indicate a transient increase of the crystal temperature, emission arising from impurities and/or defects in the crystal and a strong indication of a transient change in the absorption.

\section{EXPERIMENTAL SET-UP}

The experimental arrangement for the Raman scattering experiments consists of a Q-switched Nd:YAG laser operating at $10 \mathrm{~Hz}$, a $\mathrm{CW}$ argon laser, a single grating spectrograph and a liquid nitrogen cooled CCD detector. The 10 $\mathrm{ns}, 355 \mathrm{~nm}$ third harmonic of the YAG laser is focused to $\approx 1 \mathrm{~mm}$ diameter and it used as the pump beam illuminating the sample with $\approx 5 \mathrm{~J} / \mathrm{cm}^{2}$ along the z-axis of the crystal. The probe laser beam is the $10 \mathrm{~ns}, 532 \mathrm{~nm}$ second harmonic of the YAG laser illuminating the sample with $\approx 0.5 \mathrm{~J} / \mathrm{cm}^{2}$ with the pump pulses preceding the probe pulse by $10 \mathrm{~ns}$. The probe beam is propagating along the $\mathrm{x}$-axis of the crystal and it is focused to $\approx 100 \mu \mathrm{m}$ diameter at the crossing point with the pump beam. The pump and probe beams are overlapping inside the crystal and the Raman scattering signal from the point of overlap of the two beams is spectrally analyzed and recorded using $\mathrm{x}(\mathrm{zz}) \mathrm{y}$ scattering geometry. The samples used were fast-grown $\mathrm{KH}_{2} \mathrm{PO}_{4}$ crystals.

The laser beam utilized in the photothermal deflection and emission spectroscopy experiments is the third harmonic of a Q-switched, 3 ns pulsewidth, Nd:YAG laser. The arrangement of the photothermal deflection experiments involves overlapping the $355 \mathrm{~nm}$ pump beam focused to $\approx 30 \mu \mathrm{m}$ diameter with the $\mathrm{CW}$ beam of a diode laser focused to $\approx 85 \mu \mathrm{m}$ diameter in the bulk of the crystal at an angle of $\approx 45$ degrees. The deflection of the diode laser beam is measured using a quadrant detector and the signal is recorded using a sampling oscilloscope and a box-card integrator. The emission spectroscopy experiments were performed with the sample held at room temperature. The emitted light under $355 \mathrm{~nm}$ illumination is spectrally analyzed using a quarter meter spectrograph and recorded using a CCD detector.

\section{RAMAN SCATTERING}

Fig. 1 shows the Stokes and antiStokes Raman spectra of the $532 \mathrm{~nm}$ probe beam when the pump beam is on and when the pump beam is turned off. Three phonon peaks are observed in the $\mathrm{x}(\mathrm{zz}) \mathrm{y}$ geometry arising from the internal modes located at $363 \mathrm{~cm}^{-1}$ (v2), at $515 \mathrm{~cm}^{-1}$ (v4) and at $915 \mathrm{~cm}^{-1}$ (v1). ${ }^{5-6}$ The two spectra are overlapping except for the $915 \mathrm{~cm}^{-1}$ peak which has higher intensity (the difference in intensity is denoted as $\Delta \mathrm{I}$ ) when the pump beam is 
illuminating the sample. The increase of the $915 \mathrm{~cm}^{-1}$ peak is observed in both, the Stokes and antiStokes parts of the spectrum. The intensity of the antiStokes $363 \mathrm{~cm}^{-1}$ peak remains the same in both cases which indicates that there is no change of the crystal temperature

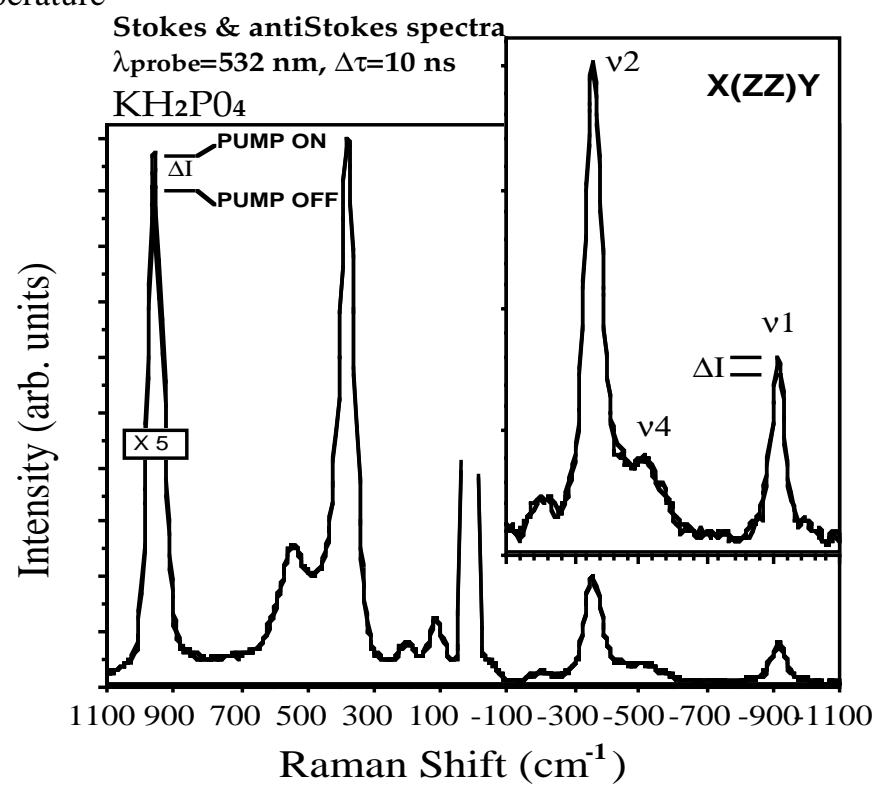

Figure 1. Stokes and antiStokes Raman spectra of the $532 \mathrm{~nm}$ probe beam when the pump beam is on and when the pump beam is turned off. The inset shows the antiStokes Raman spectrum in more detail. $\Delta \mathrm{I}$ denotes the change in intensity of the $915 \mathrm{~cm}^{-1}$ mode when the pump beam illuminated the sample.

within the experimental resolution of the Raman scattering system which is limited to approximately a few degrees. The same conclusion is reached when the ratio of the Stokes over the antiStokes intensities of the $915 \mathrm{~cm}^{-1}$ modes is used to obtain information regarding the temperature of the sample at the point where the pump and probe beams are overlapping.

Fig. 2 shows the Stokes Raman spectra of the probe beam in more detail when the pump beam is on and when it is turned off. The intensity of the of the $915 \mathrm{~cm}^{-1}$ mode is higher when the pump beam is illuminating the sample. The difference in intensity is $\Delta \mathrm{I} \approx 0.041 \mathrm{I}_{\max }$ where $\mathrm{I}_{\max }$ is the peak intensity of the $915 \mathrm{~cm}^{-1}$ line. The inset shows the digitized intensity difference spectrum after subtraction of the Raman spectrum of the probe when the pump beam is off from the Raman spectrum of the probe when the $355 \mathrm{~nm}$ pump beam is present. The peak observed in the difference spectrum is located at $915 \mathrm{~cm}^{-1}$. No additional features are observed in this spectrum. 


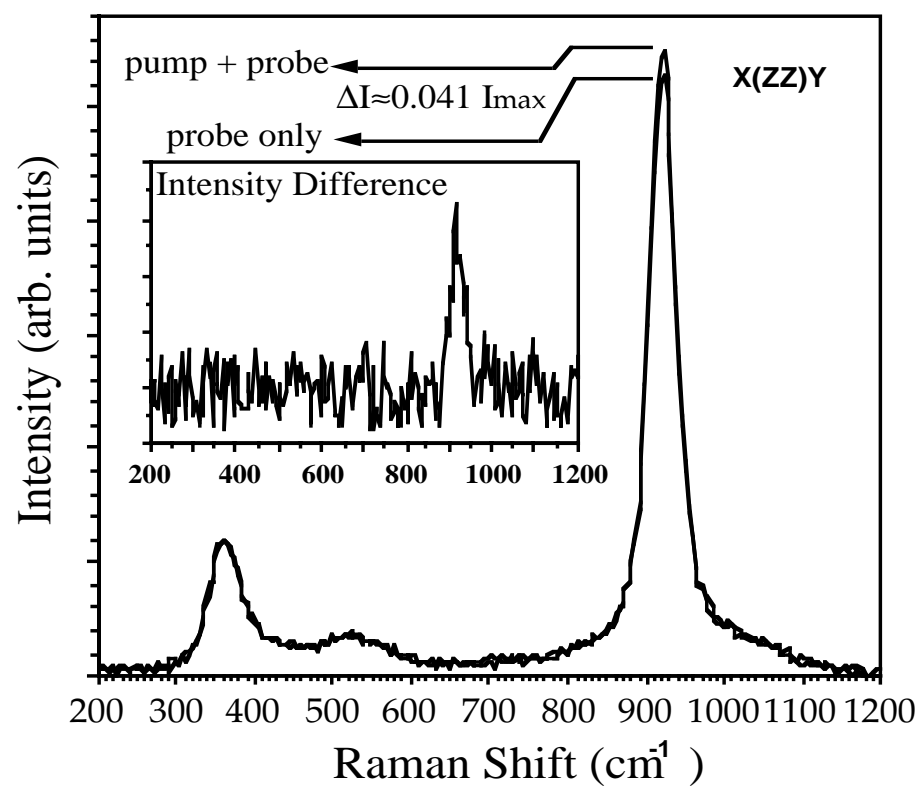

Figure 2. Stokes Raman spectra of the probe beam when the pump beam is on and when it is turned off. The inset shows the difference spectrum after subtraction of the digitized intensity profiles of the two Raman spectra.

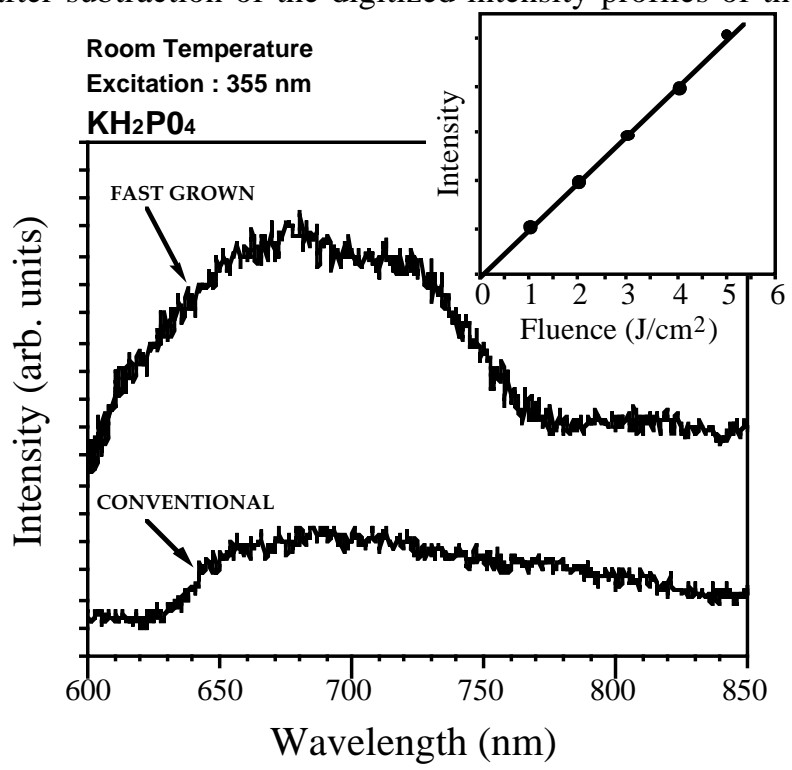

Figure 3. Emission spectra in the $600 \mathrm{~nm}$ to $850 \mathrm{~nm}$ spectral region from KDP crystals grown using the conventional and fast growth methods under $355 \mathrm{~nm}$ illumination. The inset shows the integrated intensity of the emission as a function of the laser power

\section{EMISSION UNDER 355 NM ILLUMINATION}

KDP crystals grown using the conventional and fast growth methods were illuminated with $355 \mathrm{~nm}$ laser light using below damage threshold laser fluences. Fig. 3 shows emission spectra in the $600 \mathrm{~nm}$ to $850 \mathrm{~nm}$ spectral region. The samples were held at room temperature. The change of the integrated intensity of the emission as a function of the laser power, shown in the inset of Fig. 3, demonstrates a linear dependence, indicating that the emission is due to single photon excitation of impurities in the bulk. The exact information on the composition of these impurities is not yet known. KDP samples obtained from the pyramidal sector exhibit lower emission than from the prismatic sector of the same crystal, indicating a difference in the content of impurity ions in the two sectors. Experiments to determine the damage threshold in the two sectors demonstrated practically no difference in the damage threshold. This suggests that atomic impurity ions in the crystal do not play a significant role in the damage mechanism in KDP. 


\section{PHOTOTHERMAL DEFLECTION}

The photothermal deflection technique has been proven to be a very sensitive method to measure transient variation of local temperature. ${ }^{7}$ This technique was employed to study the transient temperature change of the lattice following illumination with high power, $355 \mathrm{~nm}$, laser pulses of $3 \mathrm{~ns}$ time duration. Fig. $4 \mathrm{a}$ shows the photothermal deflection signal from the pyramidal and the prismatic sectors of the same crystal. The change in intensity may be due in part to the difference in the concentration of impurities in the two sectors and the heat deposited into the local lattice environment following the nonradiative relaxation of the photoexcited impurity ions. The decay time of the photothermal deflection signal reflects the diffusion of the transient localized heat into the rest of the crystal. Fig. 4b shows the dependence of the photothermal deflection signals on the $355 \mathrm{~nm}$ power of the laser pulse in a KDP crystal and in a light absorbing neutral density (ND) filter. This dependence is linear for the ND filter due to the single photon absorption processes associated with the heat deposited in the illuminated volume. In the KDP however, the dependence of the photothermal deflection signal on the energy of the laser pulse is not linear. This indicates multiphoton or multistep excitation processes are taking place in KDP in addition to the single photon excitation arising mainly from impurities inside the crystal.

\section{DISCUSSION}

The increase of the intensity of the $915 \mathrm{~cm}^{-1}$ mode when the high power $355 \mathrm{~nm}$ illumination beam is present may be attributed to the following possibilities: 1) the presence of stress causing a change of the Raman spectrum, 2) depolarization of the Raman scattering light and spectral component other than the A1 modes observed and, 3) change of the absorption characteristics following the pump pulse causing resonant Raman enhancement.
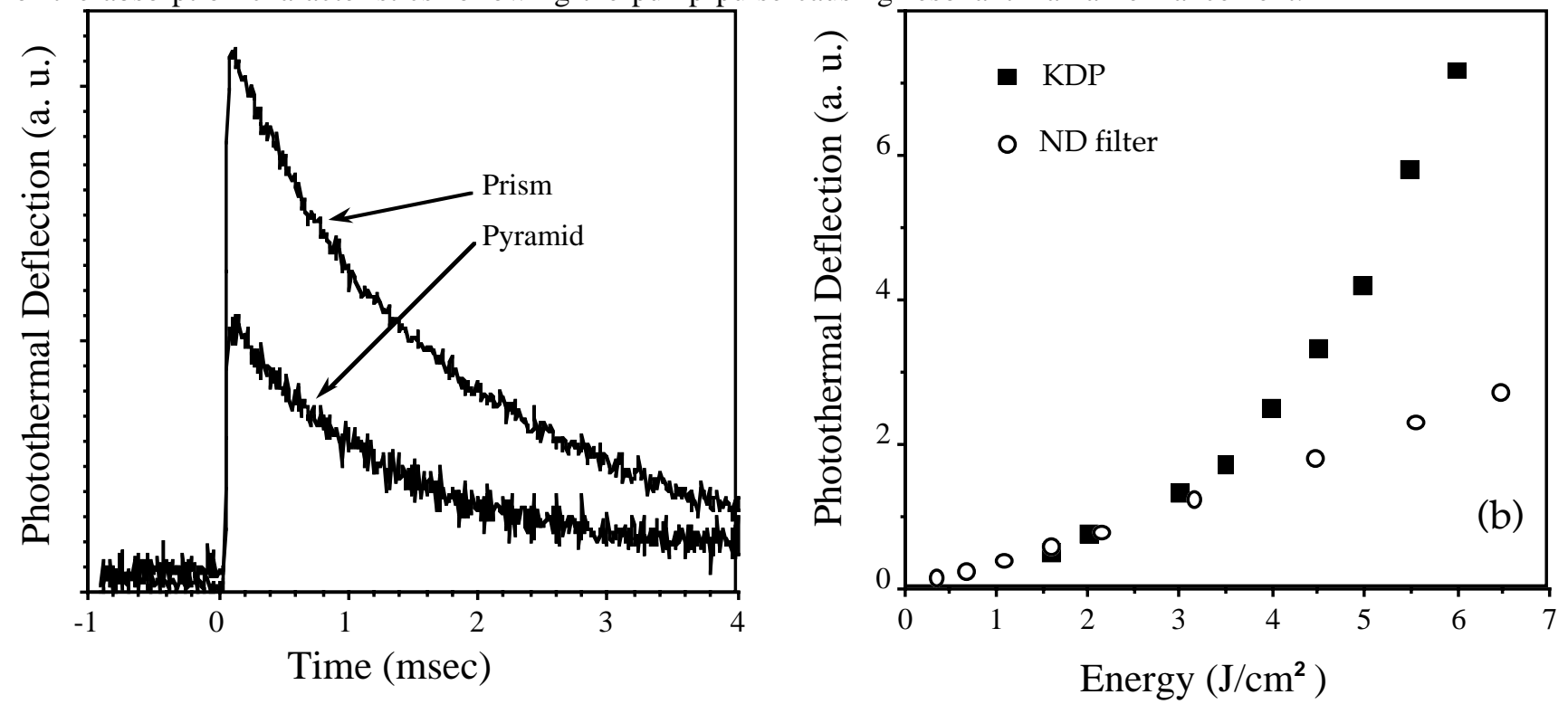

Figure 4. a) Temporal profile of the photothermal deflection signal from the pyramidal and the prismatic sectors of the same KDP crystal. b) The dependence of the photothermal deflection signals to the $355 \mathrm{~nm}$ laser power in KDP crystal and in a neutral density filter

It has been shown that when stress is applied in KDP crystals, the Raman scattering spectrum changes and shifting of the position of individual modes is observed. ${ }^{8}$ In this experiment, no shifting of the modes is observed within the experimental resolution of the system $\left(\approx 8 \mathrm{~cm}^{-1}\right)$.

If depolarization is causing the observed changes, one would expect that in addition to the A1 modes observed in the $\mathrm{x}(\mathrm{zz}) \mathrm{y}$ geometry, the spectral characteristics of the Raman spectrum in the $\mathrm{x}(\mathrm{zx}) \mathrm{y}$ geometry (the main feature of this spectrum is the dominant broad $515 \mathrm{~cm}^{-1}$ line) should become more prominent. However, this does not happen. No other alteration than the intensity change of the $915 \mathrm{~cm}^{-1}$ line in the $\mathrm{x}(\mathrm{zz}) \mathrm{y}$ Raman spectrum is observed when the 355 $\mathrm{nm}$ pulses illuminate the sample. In addition, depolarization will lower the intensity of all A1 modes rather than increasing the intensity of only one of them as is observed. 
A transient change of the absorption might be another reason for the observed changes. Absorption can be due to impurity ions (X) replacing $\mathrm{P}$ in tetrahedraly coordinated sites and due to absorption, local modes of the $\mathrm{XO}_{4}$ could be observed. However, in this case due to the difference in mass between the impurity ion and $\mathrm{P}$, the local modes should be shifted in energy with respect to the $\mathrm{PO}_{4}$ internal modes. ${ }^{9}$ This is not consistent with the experimental observations (see inset of fig. 2). The above discussion indicates that if absorption is the source of the changes in the Raman spectrum, it has to be associated with the $\mathrm{PO}_{4}$ itself. In this case, based on Albrecht's theory ${ }^{10}$, the internal modes of $\mathrm{PO}_{4}$ that should be most enhanced are the totally symmetric ones and foremost, the tetrahedral $915 \mathrm{~cm}^{-1}$ breathing mode which is the only totally symmetric mode in the $\mathrm{C}_{2}$ site symmetry that originates in a totally symmetric representation in the Td molecular symmetry. This model is consistent with the experimental results, where the 915 $\mathrm{cm}^{-1}$ mode is enhanced without any shifting in energy.

The ability of Raman scattering to measure the lattice temperature using the ratio of the intensity of the Stokes over the antiStokes lines of the same mode is limited to few degrees due to insufficient signal to noise ratio. The photothermal deflection technique is much more sensitive in detecting transient temperature variations compared to Raman scattering. The temperature-time profiles measured using the photothermal deflection technique are due to temperature variations smaller than a few degrees. The exact value of the temperature change has not been estimated. We are currently testing a system to perform a three dimensional mapping of the temperature change in KDP crystals based on the photothermal deflection technique to investigate the presence of "hot spots" inside the crystal that may be responsible for lowering the laser damage threshold. 


\section{CONCLUSION}

In conclusion, the Raman scattering, photothermal deflection and emission spectroscopy investigation of fastgrown KDP crystals under high fluence, $355 \mathrm{~nm}$ laser irradiation allowed for the investigation of physical processes taking place at below damage threshold. The Raman scattering experiment indicates that the change of the local temperature is less than few degrees while it is strongly suggestive of a transient change in the absorption. Using the photothermal deflection technique, the dynamics of the transient temperature variations indicates that in KDP the temperature increase is in part due to multistep excitation. Emission spectroscopy measurements have revealed the presence of impurities. The degree that these processes are related to the damage mechanism in KDP is subject to further investigation.

\section{ACKNOWLEDGMENTS}

This research is performed under the auspices of the U.S. Department Of Energy by Lawrence Livermore National Laboratory under contract number W-7405-ENG-48 through the Institute for Laser Science and Applications.

\section{REFERENCES}

1. N. Bloembergen, Laser-induced Electric Breakdown in Solids, IEE J. Quantum Electron. QE-10, 375, (1974).

2. S. C. Jones, P. Braunlich, R. T. Casper, X. A. Shen and P. Kelly, Recent, progress on laser-indused modifications and intrinsic bulk damage of wide-gap optical materials, Opt. Engineering, 28, 1039 (1989).

3. B. C. Stuart, M. D. Feit, S. Herman, A. M. Rubenchic, B. W. Shore and M. D. Perry, Nanosecond-to-femtosecond laser induced breakdown in dielectrics, Phys. Rev. B, 53, 1749 (1996).

4. R. M. OConnell, Onset Threshold Analysis of Defect-Driven Surface and Bulk Laser Damage, Appl. Opt., 31, 4143.(1992).

5. C. Y. She, T. W. Broberg and David F. Edwards, Raman Spectra of Tetragonal $\mathrm{KH}_{2} \mathrm{PO}_{4}$, Phys. Rev. B, 4, 1580, (1971).

6. Y. Tominaga, H. Urabe and M. Tokunaga., Internal Modes and Local Symmetry of $\mathrm{PO}_{4}$ Tetrahedrons in $\mathrm{KH}_{2} \mathrm{PO}_{4}$ by Raman Scattering, Solid State Commun. 48, 265, (1983).

7. J. F. Power., Frequency modulation time delay thermal lens effect spectrometry: a new technique of transient photothermal calorimetry, Appl. Opt., 29, 841 (1990).

8. I. Tenenaka Y. Tominaga, S. Endo and M. Kobayashi, High Pressure Raman Scattering and Local Distortion of $\mathrm{PO}_{4}$ in paraelectric $\mathrm{KH}_{2} \mathrm{PO}_{4}$, Solid State Commun. 84, 931, (1992).

9. Dana M. Calistru, W. B. Wang, V. Petricevic and R. R. Alfano, Resonance Raman Scattering in $\mathrm{Cr}^{4+}$-doped Forsterite, Phys. Rev. B, 51, 114980, (1995).

10. A. C. Albrecht, On the Theory of Raman Intensities, J. Chem. Phys., 34, 1476 (1961) 


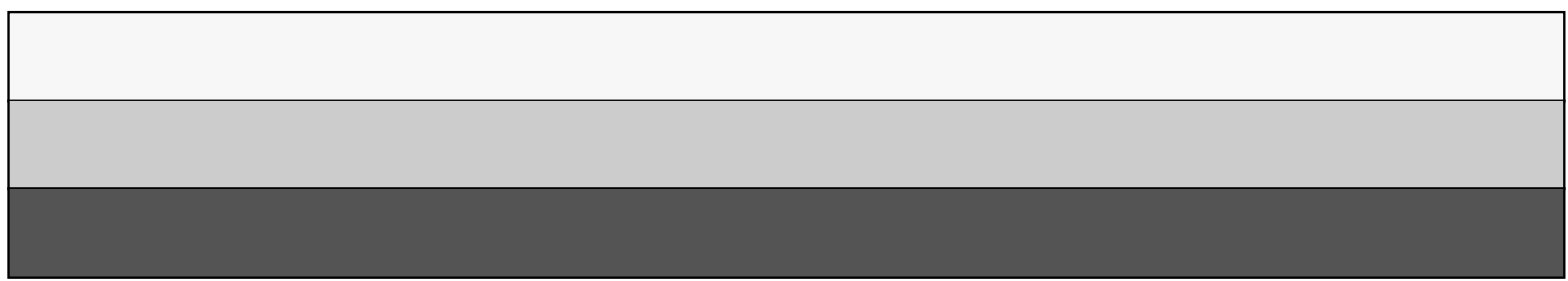

